\title{
Erweiterte Analyse ausgewählter Schwingungsphänomene mit dem C \& C2-Ansatz am Beispiel einer Einscheibentrockenkupplung
}

Peter M. Tröster, Thomas Klotz, Simon Rapp, Yulong Xiao, Sascha Ott, Albert Albers

Zwangserregtes Kupplungsrupfen ist ein Schwingungsphänomen, dessen Ursache in einer periodischen Modulation der Anpresskraft im Reibkontakt sowie des Drehmoments der Kupplung liegt. Diese periodische Modulation wird im Wesentlichen durch geometrische Abweichungen von der vorgesehenen Gestalt verursacht. Nach wie vor spielt es bei der Entwicklung von Kraftfahrzeugkupplungen eine große Rolle da die davon verursachten longitudinal Schwingungen des Fahrzeugs zu deutlichen Komforteinbußen der Fahrzeuginsassen führen. Obwohl bereits einige Einflussfaktoren des zwangserregten Kupplungsrupfens qualitativ bekannt sind, gibt es noch nicht für alle Einflussfaktoren geeignete, detaillierte Erklärungsmodelle, die Kupplungsentwicklern beim Verständnis der Wirkzusammenhänge von zwangserregtem Kupplungsrupfen unterstützen. Dies liegt unter anderem an den starken Wechselwirkungen, die über verschiedene Systemebenen auftreten und bisher kaum modelliert wurden. Daher werden in diesem Beitrag Gestalt-Funktion-Zusammenhänge auf zwangserregtes Kupplungsrupfen durch geometrische Abweichungen mithilfe des sogenannten C\& ${ }^{2}$-Ansatzes nach Albers und Matthiessen näher untersucht. Ein bereits vorhandenes Modell wird dabei um geeignete Granularitäten und Perspektiven erweitert und die Wirkzusammenhänge werden zu unterschiedlichen Zeitpunkten als sogenannte Sequenzmodelle dargestellt. In einem iterativen Prozess werden sowohl Hypothesen als auch Modelle entwickelt und es werden experimentelle Untersuchungen abgeleitet. Ausgewählte Einflussfaktoren werden hierzu in Form von Variationen an einem Prüfstand untersucht, um die Hypothesen zu verifizieren oder zu falsifizieren, und es werden erste quantitative Ergebnisse gewonnen. Dies ermöglicht die Erklärung von Ursachen für zwangserregtes Kupplungsrupfen die durch bisherige Erklärungsmodelle noch nicht hinreichend genau beschrieben werden, was durch die zum Teil großen Dynamiken der Wirkzusammenhänge begründet ist.

Keywords: Gestalt-Funktion-Zusammenhänge, Tribologie, Kupplungsrupfen, Systemmodellierung, Erklärungsmodell, Sequenzmodell 


\section{Motivation}

Während des Anfahrvorgangs kann zwangserregtes Kupplungsrupfen auftreten. Dies ist ein Schwingungsphänomen und führt zu deutlichen Komforteinbußen der Fahrzeuginsassen. Daher spielt Kupplungsrupfen bei der Entwicklung von Fahrzeug-Kupplungen nach wie vor eine große Rolle. Die Ursache dafür liegt in einer periodischen Modulation der Anpresskraft im Reibkontakt und des Drehmoments der Kupplung. Diese periodische Modulation wird unter anderem durch geometrische Abweichungen von der vorgesehenen Gestalt und der Montageposition verursacht. Liegt die Frequenz dieser Modulation im Bereich der ersten Eigenfrequenz des durch die schlupfende Kupplung dynamisch getrennten Antriebsstrangs, kommt es zu Rupfschwingungen. (Albers \& Herbst, 1998) Um Verbesserungen für die Kupplungsentwicklung ableiten zu können, müssen die Zusammenhänge und Wirkmechanismen genau verstanden werden. Bisherige Erklärungsmodelle liefern zwar eine Erklärung, welche Voraussetzungen für Rupfen vorliegen müssen, sind oft aber nicht detailliert genug, um die Wirkmechanismen beschreiben zu können. Durch die Modellierung des Wirknetzes einer Einscheibentrockenkupplung, sollen Erkenntnisse über die Anforderungen an die Modellierung von Schwingungsphänomenen gewonnen werden.

\section{Stand der Forschung}

Zunächst wird der Stand der Forschung hinsichtlich der Modellierung von GestaltFunktion-Zusammenhängen mit dem C\&C2-Ansatz beschrieben. Anschließend wird das Phänomen Kupplungsrupfen sowie Möglichkeiten zu dessen Bewertung beschrieben.

\section{Modellierung von Gestalt-Funktion-Zusammenhängen mit dem C \& C $^{2}$-Ansatz}

Für die Modellierung von Gestalt-Funktion-Zusammenhängen kann der C \& C2-Ansatz (Contact \& Channel-Ansatz) nach ALBERS und MATTHIESEN verwendet werden (Albers \& Matthiesen, 2002). Nach der Festlegung des Modellzwecks und der Definition der Systemgrenze stehen zur Modellerstellung drei Kernelemente zur Verfügung. Dazu gehören Wirkflächenpaare (WFP), Leitstützstrukturen (LSS) sowie Konnektoren (C) (siehe Abbildung 1). Während der Analyse von Gestalt-Funktion-Zusammenhängen wird nicht nur ein C\&C2-Modell erstellt, sondern es kann bei einer umfassenden Analyse zudem eine Vielzahl von Modellen aus unterschiedlichen Perspektiven, Detaillierungsgraden und 
zu unterschiedlichen Zeitpunkten erstellt werden. Dem Anwender stehen zur Beschreibung, wie systematisch auf weitere Modelle geschlossen werden kann, vier Aktivitäten zur Verfügung (Matthiesen, 2021): 1) Zoom-In und Zoom-Out: Zoom-In fokussiert die Analyse auf einen ausgewählten Ausschnitt in dem Zusammenhänge detaillierter dargestellt werden. Zoom-Out erweitert die Systemgrenze und ermöglicht somit die Analyse von Wechselwirkungen mit der Systemumgebung. Im Rahmen dieses Forschungsvorhabens wird von unterschiedlichen Systemebenen gesprochen. Zu diesen gehören das System of Systems, Supersystem, System und Subsystem (Albers et al., 2020). 2) Shift: Der Analysefokus wird auf einen anderen Aspekt im Wirknetz verschoben, wodurch benachbarte Subsysteme detaillierter analysiert werden. 3) C\&C2-Sequenzmodell: Zeitliche Veränderungen im Wirknetz werden mithilfe des Sequenzmodells dargestellt und analysiert. Dazu werden unterschiedliche Zustände mit unterschiedlichen Perspektiven und Detaillierungsgraden modelliert (vgl. (Matthiesen \& Ruckpaul, 2012)) 4) Perspektivenwechsel: Beobachtungsbarrieren werden durch eine Änderung der Betrachtungsrichtung überwunden.

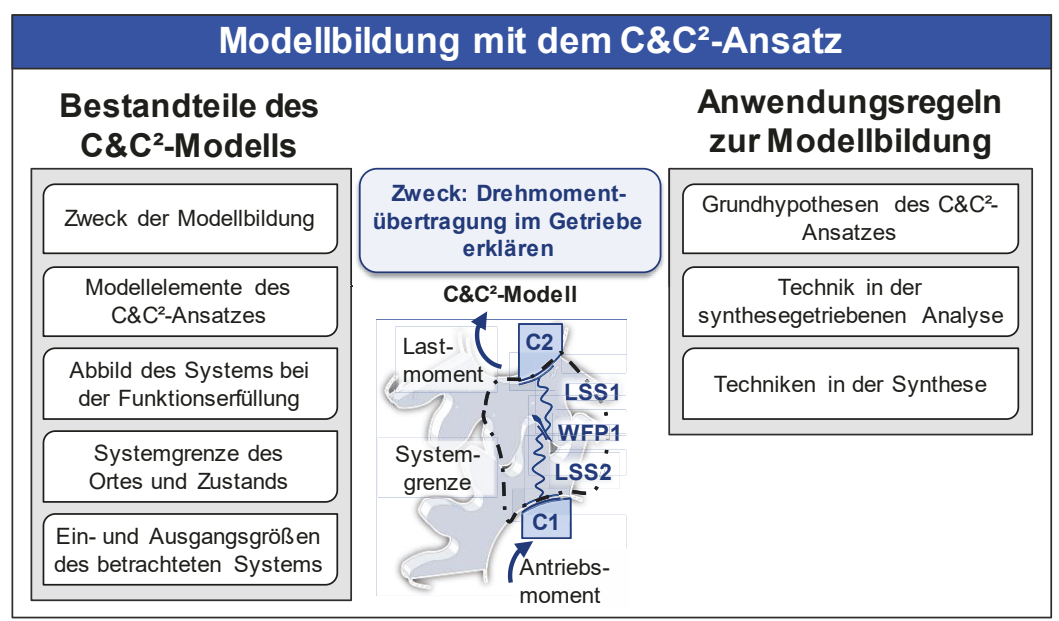

Abbildung 1: Bestandteile und Anwendungsregeln des C\&C2-Ansatzes (Matthiesen, 2021)

\section{Kupplungsrupfen und Möglichkeiten zu Bewertung und Gegenmaßnahmen}

Kupplungsrupfen bezeichnet Schwingungen im Antriebsstrang von Kraftfahrzeugen, die während der Schlupfphase beim Anfahren auftreten. Dabei treten periodische 
Wechseldrehmomente innerhalb der Kupplung auf, die den Antriebsstrang im Eigenfrequenzbereich anregen können. Durch die Räder werden diese periodischen Anregungen in longitudinale Schwingungen des Fahrzeugs übersetzt (siehe Abbildung 2 links). (Albers \& Herbst, 1998; Dresig \& Fidlin, 2014) Es wird grundsätzlich zwischen zwei Arten von Rupfen unterschieden. Selbsterregtes Rupfen kann man auf eine negative Dämpfung innerhalb des Kupplungssystems zurückführen (Krüger, 2003). Zwangserregtes Rupfen hingegen wird durch periodische Anregungen einer äußeren Energiequelle verursacht (Albers \& Herbst, 1998).

Als Gegenmaßnahmen von Rupfen können verschiedene Ansätze verfolgt werden. Dabei kann man zwischen aktiven und passiven hardware-basierten sowie aktiven software-basierten Ansätzen unterscheiden (Eppler, 2018). Unter den aktiven hardwarebasierten Ansätzen werden beispielsweise Hardware-Tilger verstanden (Hausner \& Häßler, 2012). Passive hardware-basierte Ansätze sind beispielsweise eine gezielte Optimierung der Reibflächenpaarung und der Belagfederkennlinie sowie eine kardanische Kupplungsbetätigung (Häßler \& Kessler, 2015; Hoffmann, Bührle, Kimmig, Englisch \& Götz, 2015; Kimmig, Rathke \& Reuschel, 2013). Zu den aktiven softwarebasierten Ansätzen zählen verschieden komplexe Maßnahmen, die durch eine Softwareumgebung dem Kupplungsrupfen durch eine Momentenmodulation entgegenwirken (Albers, Meid \& Ott, 2010; Eppler, 2018; Kimmig et al., 2013; Krüger, 2003; Küpper, Serebrennikov \& Göppert, 2006).

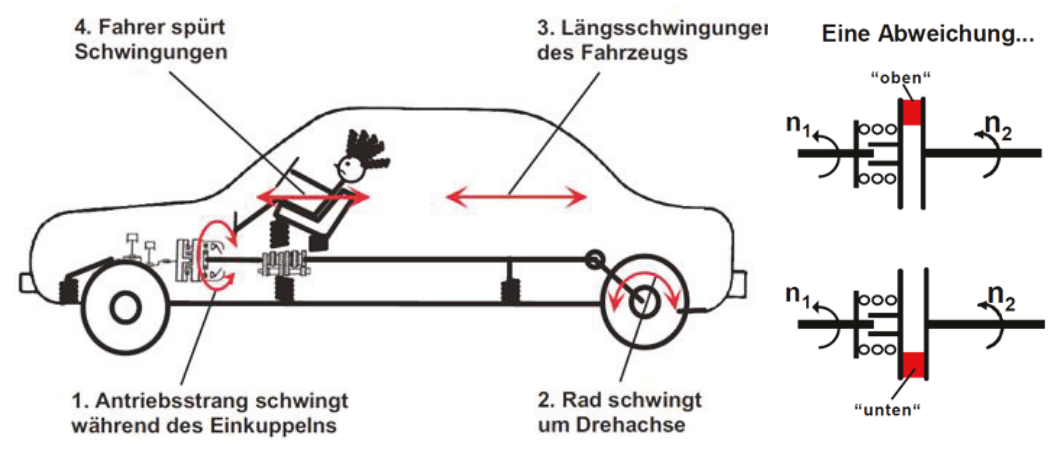

... erzeugt kein Rupfen

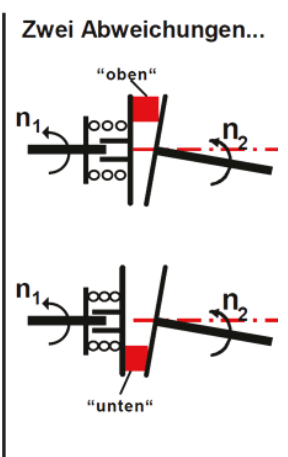

... erzeugen Rupfen

Abbildung 2: Längsschwingungen durch Kupplungsrupfen (links) und Modellbetrachtung für zwangserregte Schwingungen (rechts) (Albers \& Herbst, 1998) 
Anhand einer abstrakten Modellbetrachtung wird deutlich, dass für zwangserregtes Rupfen mindestens zwei Abweichungen gleichzeitig auftreten müssen (siehe Abbildung 2 rechts). Im Falle einer einzelnen Abweichung, die in der Abbildung als Rechteck dargestellt ist, wird die reibaktive Fläche verkleinert. Es kommt bei einer Abweichung jedoch zu keinen periodischen Drehmomentschwankungen, da sich die Abweichung mit den Reibpartnern dreht. Erst durch eine zweite Abweichung kommt es zu Schwankungen der Anpresskraft und infolge dessen zu rupfen.

Für die Bewertung von Rupfschwingungen gibt es verschiedene Möglichkeiten. In diesem Forschungsvorhaben werden neben dem Differenzdrehmoment zwei weitere Kennwerte untersucht. Zum einen wird ein Flächenindex (1) gebildet, der die Fläche zwischen Hüllkurven beschreibt. Zur Berechnung dieser Fläche wird die Differenz zwischen der oberen Hüllkurve $n(t)_{o H K}$ und der unteren Hüllkurve $n(t)_{u H K}$ über die Zeit integriert (siehe Abbildung 3). Für den Flächenindex [rad] ergibt sich folgende Berechnungsvorschrift: (Krüger, 2003)

$$
F I=\int\left(n(t)_{o H K}-n(t)_{u H K}\right) d \tau
$$

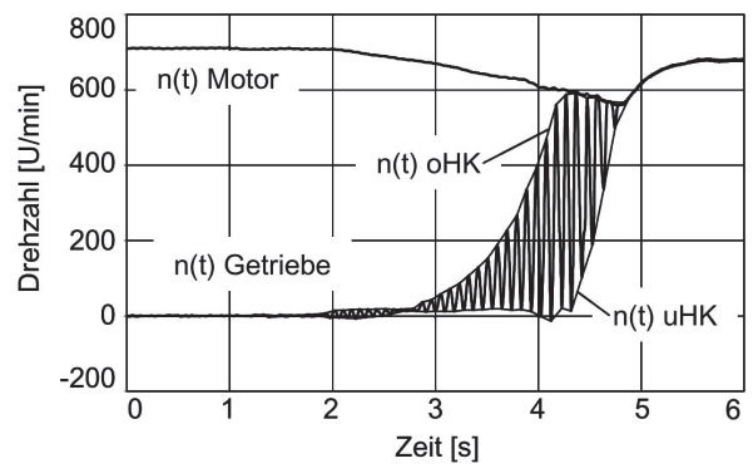

Abbildung 3: Flächenindex zur Bewertung von Schwingungen im Antriebsstrang als Fläche zwischen der oberen $n(t) o H K$ und unterer Hüllkurve $n(t) u H K$ (Krüger, 2003, S. 77)

Ein anderer genutzter Kennwert ist der Schwingungsdosiswert VDV (2) (Vibration Dose Value), da dieser einzelne Schwingungsspitzen stärker betont. Dieser Wert ist das Integral über die vierte Potenz der frequenzbewerteten momentanen Schwingbeschleunigung $a_{w}(t)$ in $\left(\frac{m}{s}\right)^{1,75} . \phi$ ist die Einwirkungsdauer der Schwingungen und in diesem Fall gleich der Messdauer T. (DIN EN ISO 8041-1) 


$$
V D V=\left(\int_{0}^{\phi} a_{w}^{4}(\xi) d \xi\right)^{\frac{1}{4}}
$$

\section{Forschungsfragen und Forschungsansatz}

\section{Forschungsbedarf und Forschungsfrage}

Es gibt bereits viele qualitativ bekannte Einflussfaktoren auf Kupplungsrupfen (Schaeffler Automotive Aftermarket GmbH \& Co. KG, 2017). Dazugehörige Erklärungsmodelle sind häufig nicht verfügbar oder nicht detailliert genug, um das Entstehen von Rupfschwingungen detailliert zu erklären. Es besteht daher der Bedarf, die detaillierten Wirkzusammenhänge für verschiedene Einflussfaktoren zu modellieren, um daraus systematisch Variationen abzuleiten und deren Einfluss mit einem Prüfaufbau zu quantisieren. Aktuell liegt in der Literatur noch wenig quantitatives Wissen über die konkreten Gestalt-Funktion-Zusammenhänge von Einflussfaktoren vor. In Forschungseinrichtungen und spezialisierten Unternehmen ist dieses Wissen teilweise in implizierter Form jedoch vorhanden. Zur systematischen Dokumentation dieses impliziten Wissens müssen die Gestalt-Funktion-Zusammenhänge der bereits bekannten Einflussfaktoren modelliert werden, um anhand der Modelle das vorhandene Wissen zu explizieren und neues Wissen zu generieren. Für die konkrete Modellierung einer Kupplung mit dem C \& $C^{2}$-Ansatz müssen dafür aber zunächst Best-Practices abgeleitet werden. Dies führt zu der Forschungsfrage:

Wie können Wirkzusammenhänge von geometrischen Einflussfaktoren auf zwangserregtes Kupplungsrupfen mit C \& $C^{2}$-Modellen erklärt werden?

\section{Forschungsansatz}

Zur Untersuchung der Wirkzusammenhänge von geometrischen Einflussfaktoren auf zwangserregtes Kupplungsrupfen wird in einem ersten Schritt eine Einscheibentrockenkupplung basierend auf einem CAD-Modell als 2D-Schnittbild in verschiedenen Systemebenen modelliert. Dieses enthält bereits bekannte und mögliche Einflussfaktoren wie geometrische Abweichungen. Ausgewählte Einflussfaktoren werden in verschiedenen Systemebenen, Detailgraden und zu verschiedenen Zeitpunkten (Sequenzmodelle) modelliert, um deren Gestalt-Funktion-Zusammenhänge zu analysieren. Darauf aufbauend werden Hypothesen gebildet, die eine logische und konsistente 
Erklärung für die jeweiligen Einflussfaktoren bieten. Im nächsten Schritt werden Möglichkeiten zur Prüfung mit einem vorhandenen Prüfaufbau abgeleitet und die Hypothesen durch Messergebnisse überprüft.

\section{Ergebnisse}

Nachfolgend wird ein initiales C \& C2-Modell mit mehreren Systemebenen vorgestellt und die dabei verwendete Bezeichnung der Systemebenen erklärt. Anschließend folgen zwei Beispiele, die mit C \& $C^{2}$-Modellen erklärt und durch Versuche mit einem Prüfaufbau quantifiziert werden.

\section{Initiales C \& C2-Modell aus mehreren Systemebenen zur Identifikation von Einflussfaktoren und Erklärung ihrer Wirkzusammenhänge}

Zur Identifikation weiterer Einflussfaktoren sowie zur Erklärung deren Wirkzusammenhänge, wurde ein initiales C \& $C^{2}$-Modell mit drei Systemebenen erstellt (siehe Abbildung 4). Ausgehend von dem in diesem Forschungsvorhaben zu untersuchenden Subsystem Kupplung (Systemebene 0) wird durch die Aktivität Zoom-Out der Antriebsstrang des Gesamtfahrzeugs in der Systemebene -1 betrachtet. Die Modellierung der angrenzenden Subsysteme entlang der Kraftflüsse ermöglicht die Analyse potenzieller Einflussfaktoren auf das Kupplungsrupfen. Zu dieser näheren Umwelt und den angrenzenden Systemen gehören unter anderem das Getriebe bis hin zu den Wirkflächenpaaren (WFPs) zwischen den Reifen und der Fahrbahn sowie die Eingaben des Fahrers. In der Abbildung sind in Ebene 0 zunächst nur die Kupplung und der Ausrücker dargestellt. Dieser wird mit der Aktivität Shift als Umgebung der Kupplung in die Untersuchung der Systemebene 0 mitberücksichtigt. In der Systemebene +1 werden schließlich die Subsysteme der Kupplung durch Zoom-In selbst modelliert und analysiert. Für jedes durch die Modellierung und Analyse des initialen C\&C²-Modells identifizierten Subsystems, wurden mögliche geometrische und physikalische Einflussfaktoren identifiziert. Im Folgenden wird dies an den zwei Beispielen Luftfeuchtigkeit im Kupplungssystem und Kombination aus Abweichung der Belagfederung und des Ausrückers exemplarisch gezeigt.

\section{Sequenzmodell für den Einflussfaktor Luftfeuchtigkeit}

Kupplungsrupfen ist ein Phänomen, welches nicht bei jeder Kupplung und bei jedem Zustand in einem Maße auftritt, dass es von den Fahrzeuginsassen negativ wahrgenommen wird. Es kann erfahrungsgemäß vermehrt bei Niederschlag und Morgentau auftreten. Dabei tritt es insbesondere bei den ersten Anfahrvorgängen auf. Es wird 
vermutet, dass diese zeitlich befristete Veränderung mit der Luftfeuchtigkeit zusammenhängt. Um mögliche Wirkzusammenhänge von Luftfeuchtigkeit auf Kupplungsrupfen zu analysieren, wurde die Friktionspaarung um zusätzliche detailliertere Modelle erweitert. Dadurch können verschiedene mögliche Veränderungen der Beschaffenheit des Reibbelags erklärt werden. Der Einflussfaktor Luftfeuchtigkeit wurde Untersuchungen unterzogen, für die eine durch eine Klimakammer genutzt wurde. Dazu wurde die Hypothese aufgestellt: Luftfeuchtigkeit erhöht die Neigung zu Kupplungsrupfen. Denn wenn die Luftfeuchtigkeit erhöht wird, dann führt dies zu einer Zunahme von Kupplungsrupfen bei den ersten Anfahrvorgängen, weil es durch die hohe Luftfeuchtigkeit zur Bildung von Kondensationswasser auf der Sekundärscheibe, der Kupplungsscheibe und der Anpressplatte kommt und dies verschiedene Effekte hervorruft. Basierend auf dieser Hypothese wurden verschiedene Modelle für eine mögliche Erklärung erstellt: Erstens: Das Kondenswasser (grün) senkt den Reibwert bei den ersten Anfahrvorgängen, indem es als Zwischenstoff die Reibung zwischen Grundkörper und Gegenkörper verringert (siehe Abbildung 4 Zustand 2.1). Diese Mischreibung führt zu einem veränderten Reibkoeffizienten der den Stip-Slick-Effekt verstärken und letztlich zu Rupfen führen kann (Maucher, 1990). Nach wenigen Anfahrvorgängen wird das komplette Kondenswasser durch Zentrifugalkräfte aus der Kupplung geschleudert.

Eine weitere mögliche Erklärung ist die schlagartige Verdampfung von Wasser auf oder im Kupplungsbelag. Dabei verkleinern die Gaskissen aus Wasserdampf die Fläche der zur Übertragung von Drehmoment genutzten WFPs und es bilden sich zusätzlich LeitStütz-Strukturen (LSS). Da beim Anfahrvorgang durch die Reibung zwischen Sekundärscheibe, Kupplungsbelag und Anpressplatte schnell Energie in das Kupplungssystem übertragen wird, führt dies lokal zu sehr starker Erhitzung. Es können sich dabei Wasserdampfkissen bilden, die zu lokal stark schwankenden Anpresskräfte und Reibwerte führen können (siehe Zustand 2.2 in Abbildung 4). Nach wenigen Anfahrvorgängen ist alles Wasser verdampft und es liegen weitestgehend die ursprünglichen Bedingungen vor.

Zweitens: Das Kondenswasser dringt in den Kupplungsbelag ein und lässt diesen quellen was zu einer Zunahme des Volumens führt. Dies ist beispielsweise dann problematisch, wenn Wasserdampf am kalten Kupplungssystem über Nacht kondensiert und sich am tiefsten Punkt der Kupplung sammelt. Dadurch kann eine lokal begrenzte Quellung entstehen, die sich wie eine Abweichung der Belagfederung oder Fertigungsfehler der Belagstärke auswirkt (vergleichbar mit Abbildung 5). Daraus resultiert beim Anfahrvorgang eine höhere Flächenpressung aufgrund der gesteigerten Belagstärke, was zu Kupplungsrupfen führen kann. 


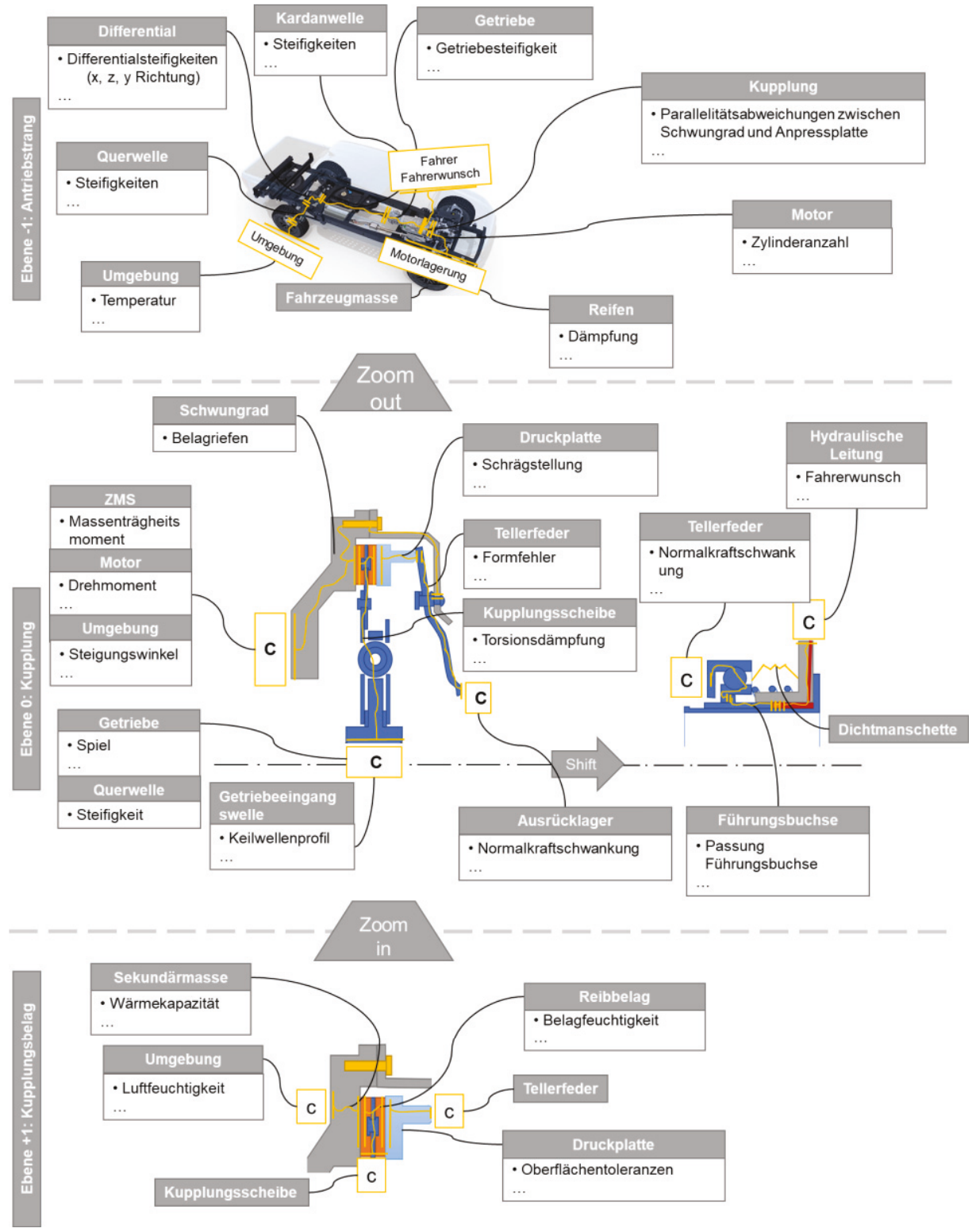

Abbildung 4: Ausschnitt des initialen und stark vereinfachten C \& C2-Modells mit drei Ebenen zur Identifikation von Wirkflächenpaaren und Konnektoren $(C)$ und bekannten Einflussfaktoren - Bild in Ebene -1 (Ford Vehicles, 2021) 


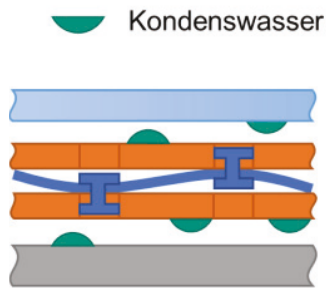

Zustand 1

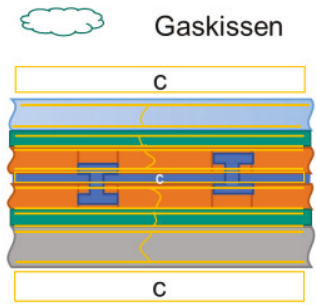

Zustand 2.1

LSS $\quad$ WFP

c Konnektor

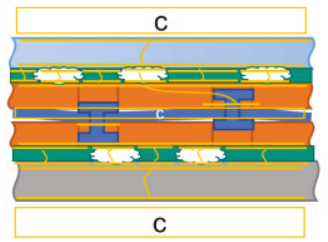

Zustand 2.2

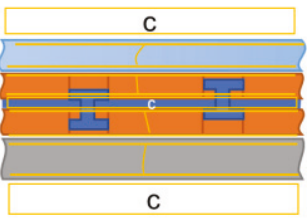

Zustand 3

Abbildung 5: Sequenzmodell zur Erklärung von Rupfen durch die Bildung von Kondenswasser zwischen den Reibpartnern und die Entstehung von Wasserdampf, die zu lokal verschiedener Drehmomentübertragung führen

Zur Überprüfung der Luftfeuchtigkeitshypothese wurden zunächst mehrere Anfahrzyklen bei $5 \%$ iger Luftfeuchtigkeit und $60^{\circ} \mathrm{C}$ durchgeführt. Diese Werte wurden durch eine Klimakammer über alle Anfahrzyklen konstant gehalten. Die Messergebnisse zeigen, dass bei $50 \%$ iger Luftfeuchtigkeit und $60^{\circ} \mathrm{C}$, die über einen Zeitraum von zwei Stunden auf das Kupplungssystem eingewirkt haben die ersten beiden Anfahrzyklen deutlich höhere Drehmomentdifferenzen aufweisen (Abbildung 6 ganz rechts).
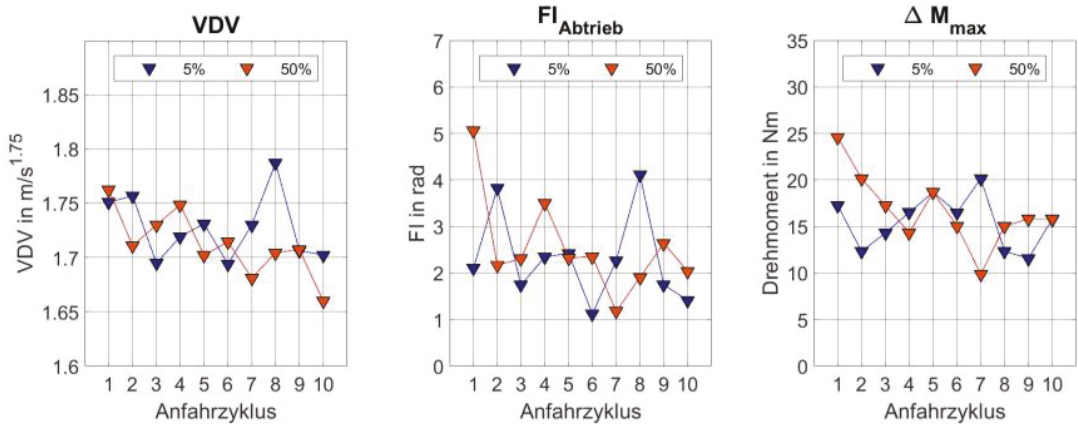

Abbildung 6: Berechnete Flächenindizes und gemessene Differenzdrehmoment bei verschiedener Luftfeuchtigkeit im Verlauf mehrerer Anfahrzyklen verdeutlichen den Einfluss der Luftfeuchtigkeit auf die Neigung zu Kupplungsrupfen in den ersten Anfahrvorgängen

Zur Analyse des Effekts des Quellverhaltens des Kupplungsbelags wurde die Kupplungsscheibe zunächst für $1,5 \mathrm{~h}$ auf $150^{\circ} \mathrm{C}$ erhitzt und dabei getrocknet. Nach dieser Behandlung wurde die Masse der Kupplungsscheibe mit 2420,56 g und die Dicke der Kupplungsscheibe mit 9,217 mm gemessen. Vor der Messung zu einem zweiten aufgequollenen Zustand wurde die Kupplungsscheibe mit destilliertem Wasser benetzt, 
in Folie eingewickelt und 1,5 h bei Raumtemperatur gelagert. Anschließend wurde die Masse mit 2425,08 g und die Dicke mit 9,33 mm gemessen. Es ergibt sich eine Massendifferenz von 4,523 g und eine Differenz der Dicke um 0,117 mm der Kupplungsscheibe. Dies bedeutet eine Zunahme der Masse um 0,17\% und Dicke um 1,27 \% durch infolge der Luftfeuchtigkeit. In den ersten zwei Anfahrvorgängen wirkt sich dies durch eine Steigerung der Drehmomentdifferenz um 45 \%-65 \% aus.

\section{Sequenzmodell zu Abweichungen der Belagfederung bei gleichzeitigem Zungenschlag}

Als zweites Beispiel wird der Wirkzusammenhang zwischen einer Abweichung der Kupplungsscheibe und einer ungleichmäßig abgehobenen Anpressplatte modelliert. Diese Abweichungen können durch verschiedene Konstellationen verursacht werden. Eine Abweichung der Belagfederung kann wie eine Abweichung der Belagstärke wirken. Dies ist im Modell stark vereinfacht als zusätzlicher Teilkreis mit abgeflachten Kanten modelliert (siehe Abbildung 7). Die ungleichmäßig abgehobene Anpressplatte wird durch eine angewinkelte Kreisscheibe modelliert.

Kupplungsscheibe
mit geometrischer
Abweichung im
Reibbelag
verursacht durch
Abweichung der
Belagfederung
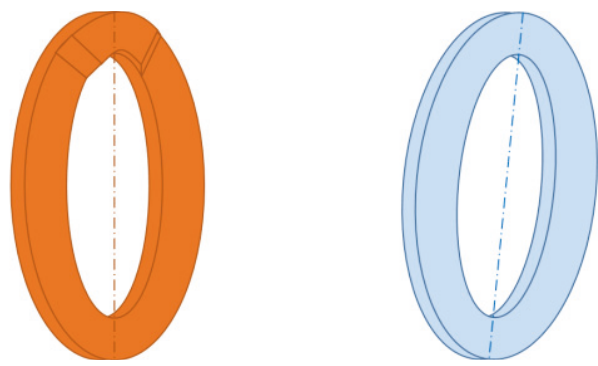

Ungleichmäßig abgehobene Anpressplatte

Abbildung 7: Modell einer geometrischen Abweichung im Reibbelag und gleichzeitig ungleichmäßig abgehobener Anpressplatte

Beim Anfahrvorgang führen die beiden Abweichungen aufgrund der Differenzdrehzahl zu zyklisch auftretenden Schwankungen der lokalen Flächenpressung (siehe Abbildung 8 In diesem Modell ist die Anpressplatte aus Gründen der einfacheren Modellierung statisch dargestellt. Im realen Kupplungssystem steht die Kupplungsscheibe beim Anfahrvorgang bis zum ersten Kontakt mit der sich drehenden Anpressplatte.

Die lokale Flächenpressung steigt beim Zusammentreffen der beiden Abweichungen an und führt zu einem kurzfristigen Anstieg des übertragenen Drehmoments. Mit einem C \& C²-Modell lässt sich dies durch die Ausbildung zusätzlicher WFPs erklären, die sich beim Zusammentreffen zweier Abweichungen ausbilden (siehe Abbildung 9). 
Diese sorgen für eine größere Fläche, die zur Übertragung von Drehmoment genutzt werden kann. Zur Überprüfung der Hypothese wurde die Belagfederung an einer Stelle durch das Unterlegen mit 0,5 mm Unterlegfolie manipuliert (siehe Abbildung 10 links). Die ungleichmäßig abgehobene Anpressplatte wurde durch Unterlegen des Ausrücklagers mit 0,5mm Unterlegfolie und einem Abtrag der Zungenauflage um 1,5mm erzeugt (siehe Abbildung 10 rechts).

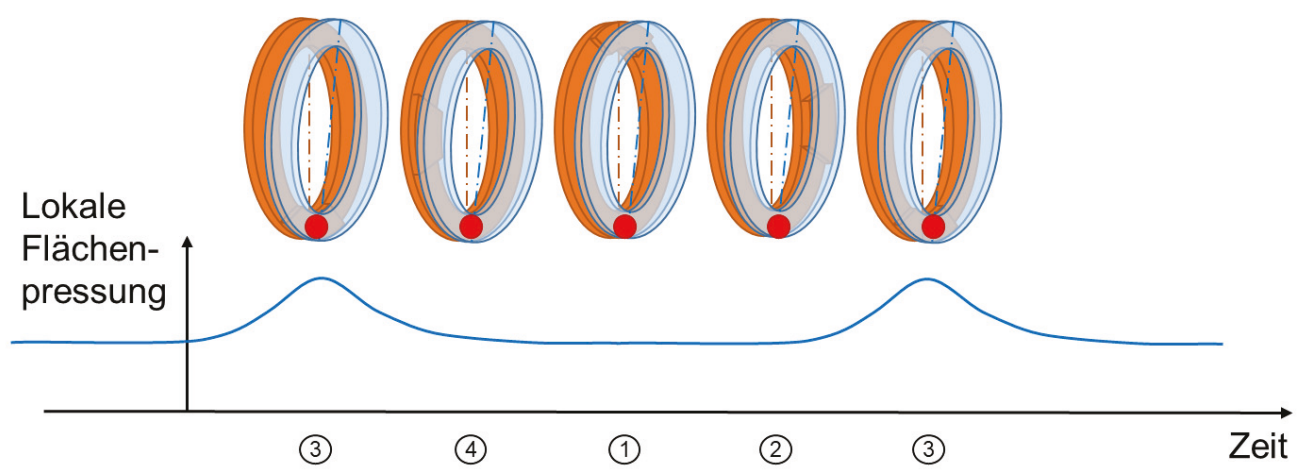

Abbildung 8: Qualitativer Verlauf der lokalen Flächenpressung am markierten Punkt während der relativen Drehung der Kupplungsscheibe mit der Abweichung um die Anpressplatte
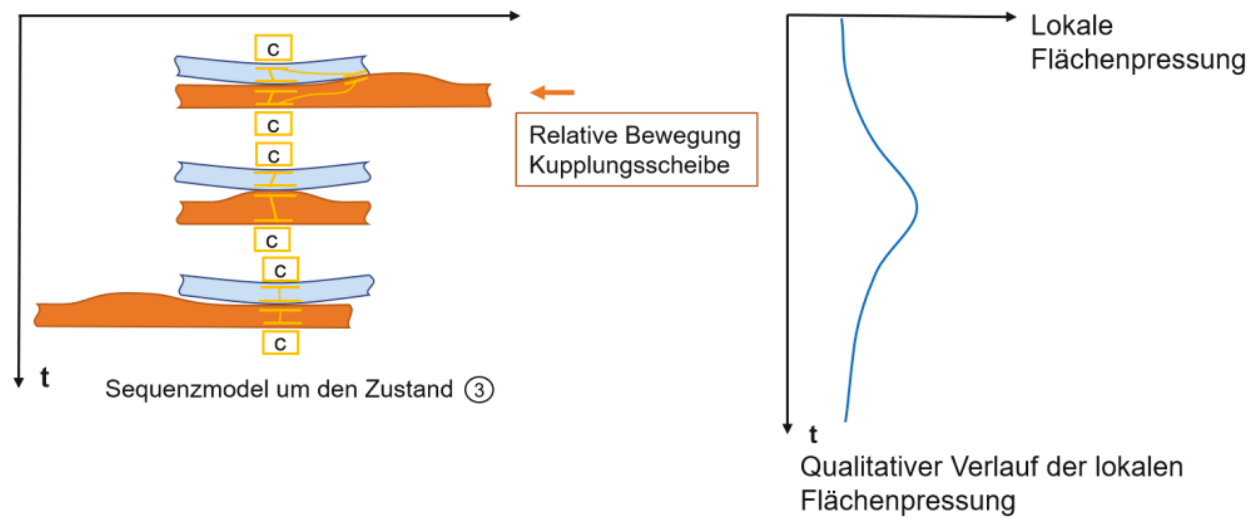

Abbildung 9: Sequenzmodell und qualitativer Verlauf der lokalen Flächenpressung beim Aufeinandertreffen zweier geometrischer Abweichungen 

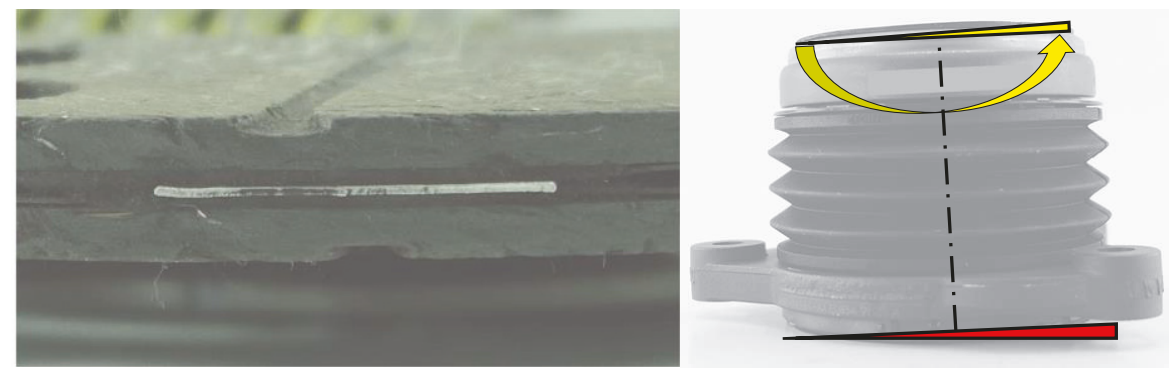

Abbildung 10: Links: Manipulierte Kupplungsscheibe mit 0,5mm Unterlegfolie zwischen den Federblechen Rechts: Manipuliertes Ausrücklager. Höhenschlag durch $1 \mathrm{~mm}$ Unterlegfolie (unten) und Abtrag der Zungenauflage um 1,5mm (oben)

Der erste Versuch zeigt einen nahezu rupffreien Anfahrvorgang. Das zweite Messergebnis zeigt die Auswirkungen der manipulierten Belagfederung. Der Flächenindex des Getriebes steigt dadurch um über $550 \%$, der des Abtriebs um über $750 \%$, die Drehmomentdifferenz um etwa $345 \%$ sowie der VDV um etwas mehr als $5 \%$. Weitere Messergebnisse werden in Tabelle 1aufgeführt.

Tabelle 1: Kennzahlen für verschiedene Konfigurationen des Prüfaufbaus

\begin{tabular}{lllll} 
& $\begin{array}{l}\text { Ohne künstliche } \\
\text { Fehler }\end{array}$ & $\begin{array}{l}\text { Manipulierte Belag- } \\
\text { federung }\end{array}$ & $\begin{array}{l}\text { Manipuliertes } \\
\text { Ausrücklager }\end{array}$ & $\begin{array}{l}\text { Manipulierte } \\
\text { Belagfederung } \\
\text { und Ausrücklager }\end{array}$ \\
\hline$F I_{\text {Getriebe }}[\mathrm{rad}]$ & $\mathbf{4 , 4 8}$ & $\mathbf{3 3 , 3 3}$ & $\mathbf{5 , 9 1}$ & $\mathbf{2 7 , 5 9}$ \\
$F I_{\text {Abtrieb }}[\mathrm{rad}]$ & $\mathbf{1 , 6 6}$ & $\mathbf{1 4 , 9 0}$ & $\mathbf{1 , 7 6}$ & $\mathbf{1 1 , 1 7}$ \\
$\Delta M_{\text {max }}[\mathrm{Nm}]$ & $\mathbf{1 7 , 2 4}$ & $\mathbf{8 5 , 1 7}$ & $\mathbf{2 1 , 8 2}$ & $\mathbf{6 7 , 7 3}$ \\
$V D V\left[\frac{\mathrm{m}}{\mathrm{s}}{ }^{-75}\right]$ & $\mathbf{1 , 7 2}$ & $\mathbf{1 , 8 4}$ & $\mathbf{1 , 7 1}$ & $\mathbf{1 , 7 9}$ \\
\hline
\end{tabular}

\section{Diskussion}

Zunächst werden die ursprünglich verwendete Bezeichnung der Systemebenen sowie die im Paper verwendete Bezeichnung diskutiert. Die im Ergebniskapitel vorgestellten Beispiele werden bezüglich ihrer Modellierung und den aus den Modellen ableitbaren Möglichkeiten für Optimierungen reflektiert. Mit einem Fazit werden die wichtigsten Punkte zusammengefasst. 


\section{Initiales C \& C2-Modell aus mehreren Ebenen zur Identifikation von Einflussfaktoren und Erklärung ihrer Wirkzusammenhänge}

Zu Beginn des Forschungsvorhaben wurden die Betrachtungsebenen ausgehend vom Gesamtfahrzeug definiert. So wurde das Gesamtfahrzeug als Systemebene 1, die Kupplung als Systemebene 2 und die Friktionspaarung als Systemebene 3 bezeichnet. Zur Erarbeitung eines Leitfadens, auf was dieses Forschungsvorhaben abzielt, scheint es sinnvoller die Systemebenen ausgehend vom zu untersuchenden Gegenstand zu definieren. Daher wird die Systemebene der Kupplung als Gegenstand der Untersuchung als Systemebene 0 definiert (siehe Abbildung 4). Die Aktivität Zoom-In führt infolge bei jeder Ausführung zur Bezeichnung ${ }_{1}+1{ }^{\prime \prime}{ }_{1}{ }^{2}{ }^{\prime \prime}$ und so weiter und die Zoom-Out entsprechend $z u,{ }_{n}-1{ }^{\prime \prime},{ }_{n}-2^{\prime \prime}$. Diese Neudefinition der Nomenklatur der Betrachtungsebenen soll dem Betrachter helfen, schneller zu begreifen, auf welcher Systemebene er sich relativ zum untersuchten Subsystem im Modell befindet. Zusätzlich soll die angepasste Nomenklatur die Erstellung eines für verschiedene Systeme gültigen Leitfadens vereinfachen, da die Ebenen-Definition vereinheitlicht wird.

\section{Einfluss der Luftfeuchtigkeit auf Kupplungsrupfen}

Das Beispiel der Luftfeuchtigkeit zeigt, dass selbst bei maßgenauer Fertigung und korrektem Einbau, der im Forschungsvorhaben durch Laserausmessungen sichergestellt wurde, Umwelteinflüsse das Anfahrverhalten deutlich beeinflussen können. Die Autoren weißen darauf hin, dass keines der C \& $C^{2}$-Modelle die alleinige Erklärung für den in den Versuchen nachgewiesenen Einfluss der Luftfeuchtigkeit ist. Vielmehr treten diese Effekte in Superposition auf und eine genaue Unterscheidung der jeweiligen Anteile erscheint schwierig. Eine Möglichkeit zur präventiven Reduktion des Einflusses der Luftfeuchtigkeit könnte deren Reduktion durch zum Beispiel eine verbesserte Abdichtung und Entfeuchtung der Getriebeglocke sein. Für automatische Schaltungen könnte eine Anti-Rupf-Regelung vor dem gewünschten Anfahrvorgang die Kupplung bei betätigten Bremsen teilschließen, um Kondenswasser ab zu schleudern oder zu verdampfen. Dadurch würde rupfarmes Anfahren trotz vorheriger Feuchtigkeitseinwirkung möglich werden.

\section{Abweichungen der Belagfederung bei gleichzeitigem Zungenschlag}

Verschiedene Konstellationen von Abweichungen können zu ähnlichen Auswirkungen an der Friktionspaarung (z. B. statt Zungenschlag schiefes Ausrücklager) führen. Dieses Modell kann daher als Grundlage genutzt werden, um weitere Einflussfaktoren zu erklären. Interessant wird dabei die Untersuchung der Gemeinsamkeiten und Unterschiede verschiedener Einflussfaktoren in einem Modell höherer Detaillierung. Es 
zeigte sich, dass selbst kleine einzeln manipulierte Abweichungen einen sehr starken Einfluss auf die Neigung zum Rupfen haben. Da nach dem Modell von AlBers und HERBST immer mindestens zwei Abweichungen auftreten müssen wird deutlich, dass trotz sorgfältiger Montage bereits kleine Abweichungen von der vorgesehenen Gestalt vorhanden sein müssen (Albers \& Herbst, 1998). Die Wechselwirkungen zwischen sehr kleinen Abweichungen und dem Rupfen stellen einen spannenden Forschungsgegenstand dar. Die Darstellung von zusätzlichen WFPs ist im Beitrag idealisiert. In der Realität ist es durchaus möglich, dass sich keine zusätzlichen WFPs bilden, sondern aufgrund von Elastizitäten die zur Drehmomentübertragung zur Verfügung stehenden Wirkflächen (WF) vergrößern.

\section{Fazit}

In diesem Beitrag werden zwei initiale Beispiele detaillierterer Sequenzmodelle mit dem C \& C2-Ansatz auf verschiedenen Systemebenen gezeigt. Diese ermöglichen die Erklärung von Ursachen für zwangserregtes Kupplungsrupfen, die durch bisherige Erklärungsmodelle noch nicht hinreichend genau beschrieben werden was durch die zum Teil großen Dynamiken der Wirkzusammenhänge begründet ist. Es zeigt sich, dass C \& C²-Modelle in unterschiedlichen Systemebenen und Granularitäten bei der Abfrage und Explizierung von implizitem Expertenwissen entlang des Wirknetztes unterstützen. Dadurch lassen sich Explizierungslücken im Stand der Forschung, der auf wenige öffentliche Ursprungsquellen zurückgreift, nach und nach systematisch schließen. Darüber hinaus wird durch die C \& C2-Modellierung eine systematische Ableitung von Variationen im Kupplungssystem ermöglicht, die für die Quantifizierung der identifizierten Einflussfaktoren notwendig ist.

\section{Ausblick}

Im weiteren Verlauf des Forschungsvorhabens sollen weitere Einflussfaktoren modelliert und im Anschluss quantifiziert werden. Dazu ist es neben einem weiterentwickelten Prüfaufbau notwendig bei der Modellierung noch weiter ins Detail zu gehen und besonders die Wechselwirkungen zwischen und innerhalb der Systemebenen genauer zu beschreiben. Dafür muss der C \& C2-Ansatz voraussichtlich weiterentwickelt werden. Innerhalb der Kupplung können verschiedene Abweichungen eine ähnliche Konstellation verursachen, die sich wie in Abbildung 10 auf das Kupplungsrupfen auswirkt. Es wird daher ein methodischer Ansatz benötigt, mit dem diese Konstellationen systematisch modelliert und erkannt werden. Dadurch ließen sich kritische und unkritische Abweichungen definieren sowie Hinweise für konstruktive Verbesserungen der Kupp- 
lung ableiten. Dieser methodische Ansatz soll zur Unterstützung von Kupplungsentwicklern bei der Identifikation und Quantifizierung von Einflussfaktoren in einem Leitfaden für die Modellierung von Gestalt-Funktion-Modellen mit dem C\&C2-Ansatz münden.

\section{Danksagung}

Die Autoren danken für die Unterstützung des Forschungsprojekts „Erarbeitung einer Methodik zur Ableitung von Variationen in der PGE auf der Basis teilquantifizierter Gestalt-Funktion-Modelle am Beispiel zwangserregten Kupplungsrupfens" - AL 533/39-1 mit der Projektnummer 416374536 durch die Förderung der Deutschen Forschungsgemeinschaft (DFG). Zudem bedanken sich die Autoren bei der Ford-Werke GmbH für die Unterstützung und die zur Verfügung gestellte Teile des Antriebsstrangs, mit denen die Versuche gefahren wurden.

\section{Literaturverzeichnis}

Albers, A., Meid, M. \& Ott, S. (2010): Avoiding clutch excited judder by using an active clamping force control. In: ISMA2010 Albers, A. \& Herbst, D. (1998): Kupplungsrupfen - Ursachen, Modellbildung und Gegenmaßnahmen. In: Schwingungen in Antrieben, 98. Tagung Frankenthal (VDI-Berichte, Bd. 1416). Düsseldorf: VDI-Verlag.

Albers, A., Hirschter, T., Fahl, J., Woehrle, G., Reinemann, J. \& Rapp, S. (2020): Generic Reference Product Model for Specifying Complex Products by the Example of the Automotive Industry. In: Digital Proceedings of TMCE 2020, 353-370.

Albers, A. \& Matthiesen, S. (2002): Konstruktionsmethodisches Grundmodell zum Zusammenhang von Funktion und Gestalt technischer Systeme. In: Konstruktion Zeitschrift für Produktentwicklung, 55-60.

DIN EN ISO, 8041-1: Schwingungseinwirkung auf den Menschen - Messeinrichtung. Berlin: Beuth Verlag GmbH.

Dresig, H. \& Fidlin, A. (2014): Schwingungen mechanischer Antriebssysteme. Berlin, Heidelberg: Springer Berlin Heidelberg.

Eppler, F. (2018): Entwicklung von Methoden zum Entwurf einer robusten Anti-Rupf-Regelung am Beispiel von Fahrzeugen mit trocken laufender Doppelkupplung. In Forschungsberichte. Karlsruher Institut für Technologie (KIT), Karlsruhe.

Ford Vehicles. (2021): Ford Ranger Raptor. Beitrag im Internet: https://www.pacificford.co.nz/Page/103/ranger-raptor, abgerufen am 24.03.2021.

Häßler, M. \& Kessler, M. (2015): Entwicklungsbegleitende Simulationsmethoden zur Vermeidung von Anfahrrupfen. In: Fachtagung Kupplungen und Kupplungssysteme in Antrieben. Düsseldorf: VDI Wissensforum.

Hausner, M. \& Häßler, M. (2012): Kupplungsscheibe mit Frequenztilger gegen Rupfschwingungen. In: Automobiltechnische Zeitschrift, 64-69.

Hoffmann, J., Bührle, P., Kimmig, K.- L., Englisch, A. \& Götz, A. (2015): Double Clutch Systems - comfortable, efficient, sporty. In: Internationaler VDI-Kongress Getriebe in Fahrzeugen - Drivetrain for Vehicles. Düsseldorf: VDI Wissensforum. 
Kimmig, K.- L., Rathke, G. \& Reuschel, M. (2013): The Next Generation of Efficient Dry Double Clutch-Systems In: Internationaler VDI-Kongress Getriebe in Fahrzeugen - Drivetrain for Vehicles. Düsseldorf: VDI Wissensforum

Krüger, A. (2003): Kupplungsrupfen - Ursachen, Einflüsse und Gegenmaßnahmen. Dissertation. Universität Karlsruhe, Karlsruhe. Küpper, K., Serebrennikov, B. \& Göppert, G. (2006): Software für automatisierte Getriebe In: LuK forward. Bühl.

Matthiesen, S. (2021): Prozess und Methoden der Gestaltung. In B. Bender \& K. Gericke (Hrsg.), Pahl/Beitz Konstruktionslehre. Methoden und Anwendung erfolgreicher Produktentwicklung (9. Auflage 2021). Berlin, Heidelberg: Springer-Verlag GmbH. Matthiesen, S. \& Ruckpaul, A. (2012): New Insights on the Contact\&Channel-Approach - Modelling of Systems with several logical states. In: International Design Conference - Design 2012, 2012.

Maucher, P. (1990): Kupplungsrupfen, Möglichkeiten zur Vermeidung. In: LuK-Kolloquium, 103-117.

Schaeffler Automotive Aftermarket GmbH \& Co. KG (2017): LuK Schadensdiagnose. Leitfaden für die Beurteilung von Störungen am Kupplungssystem. Beitrag im Internet: https://www.repxpert.de/de/mediadocument/LuK-TecBr-Clutch-Failurediagnosis-PC/de, abgerufen am 17.12.2020.

\section{Kontakt}

Peter M. Tröster, M. Sc.

+4972160847252

peter.troester@kit.edu

Karlsruher Institut für Technologie (KIT)

IPEK - Institut für Produktentwicklung

Kaiserstraße 10

76131 Karlsruhe

www.ipek.kit.edu 